\title{
ION CYCLOTRON RESONANCE HEATING ON TEXTOR
}

\author{
R. KOCH,* P. DUMORTIER, F. DURODIÉ, S. HUYGEN, A. LYSSOIVAN, A. M. MESSIAEN, \\ P. E. VANDENPLAS, G. VAN WASSENHOVE, M. VERVIER, and R. R. WEYNANTS \\ Laboratory for Plasma Physics, Ecole Royale Militaire - Koninklijke Militaire School \\ Association EURATOM-Belgian State, Trilateral Euregio Cluster, B-1000 Brussels, Belgium
}

Received April 29, 2004

Accepted for Publication September 11, 2004

The original work done on TEXTOR on ion cyclotron resonance heating of the plasma is reviewed. After a brief introduction outlining the principles, the radio-frequency (rf) system is described, with its substantial evolution during time. Then, the different physics results are reviewed. Ion cyclotron heating has been performed in a large number of scenarios and under a wide range of conditions. Aside from the various minority or modeconversion scenarios, the interaction with beam ions and the possibility of controlling fast-ion diffusion with rf have been deeply investigated. Both the interaction with the wall or edge plasma and the impact on improved core confinement were studied in detail. Pioneering work was done to demonstrate efficient heating with unshielded antennas and plasma production in a tokamak by rf alone for plasma startup assistance or wall conditioning.

KEYWORDS: tokamak, ion-cyclotron heating, radio-frequency system

\section{THE HEATING OF PLASMAS IN THE ION CYCLOTRON FREQUENCY RANGE}

The principle of ion cyclotron resonance heating (ICRH) is to launch in the plasma a wave in which the electric field is rotating at exactly the same angular frequency as that of the ions of the plasma, which gyrate around magnetic field lines at the cyclotron frequency $\omega_{c i}=Z_{i} e B / m_{i}$. Here, $Z_{i}, Z_{i} e$, and $m_{i}$ are, respectively, the atomic number, electric charge, and mass of the ions of species $i$, and $B$ is the magnetic induction. As the magnetic field in a tokamak varies inversely with major radius $R, \omega_{c i}$ varies with radius, and the resonance occurs

\footnotetext{
*E-mail: r.koch@fz-juelich.de
}

only at a specific major radius $R_{r}$ where the angular frequency of the applied wave $\omega$ exactly equals the local cyclotron frequency

$$
\omega=\omega_{c i}\left(R_{r}\right) .
$$

This locus is a vertical line in the plasma cross section called the cyclotron layer. An example is shown in Fig. 1, where the axis of the machine is on the left. The magnetic field is higher on the left side of the cross section [called the high-field side (HFS)] than on the right side [called the low-field side (LFS)]. Two cyclotron layers are shown in Fig. 1. The vertical line on the right is the hydrogen cyclotron layer $\left(f=f_{c H}\right.$, where $f=\omega / 2 \pi$ is the frequency). That on the left is the six-time ionized oxygen $\left(\right.$ or $\mathrm{O}^{6+}$ ) cyclotron layer. The wave that propagates across the plasma in the ion cyclotron frequency range (ICRF) is called the fast wave (FW) and is evanescent in the low-density and vacuum region surrounding the plasma (where the antenna is located). Therefore, the distance between the antenna and the outer cutoff, where the FW starts propagating, is an important parameter that has an impact on the coupling capability of the antenna, i.e., the amount of power coupled to the plasma at a given antenna voltage.

Unfortunately, as it approaches the cyclotron layer, the FW gets more and more right-hand circularly polarized (a component that rotates opposite to the ions). The ratio of the left-polarized $\left(E_{L}\right)$ to the right-polarized $\left(E_{R}\right)$ components is given, ${ }^{1-3}$ as the wave approaches the resonance layer, by

$$
\frac{E_{L}}{E_{R}} \approx \frac{\omega-\omega_{c i}}{\omega+\omega_{c i}} .
$$

This means that at the resonance, the electric field rotates in the direction opposite to the cyclotron rotation of ions, and therefore, the wave cannot resonate with their motion and heat them. To cope with this situation, a widely used heating scheme is minority heating. For example, if one wishes to heat deuterium plasma, one can introduce a small minority of hydrogen into the deuterium and heat it at its cyclotron angular frequency $\omega=\omega_{c H}=2 \omega_{c D}$. 


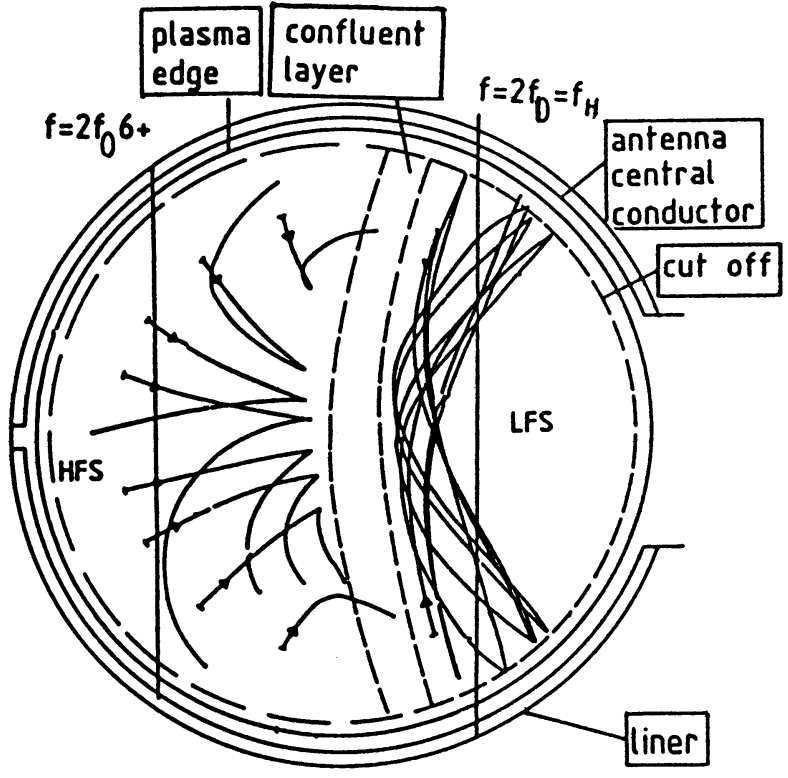

Fig. 1. Cross section of TEXTOR showing the liner, the position of the antenna's central conductors (also called "straps"), the outer cutoff of the fast wave (circular dashed line), the confluent layer between the resonance (left dashed line) and cut-off (right dashed line), and wave rays (solid lines). ${ }^{4}$

As the polarization is determined by the majority species (D),

$$
\frac{E_{L}}{E_{R}} \approx \frac{\omega-\omega_{c D}}{\omega+\omega_{c D}}=\frac{2 \omega_{c D}-\omega_{c D}}{2 \omega_{c D}+\omega_{c D}}=\frac{1}{3} .
$$

There is now a substantial fraction of the electric field rotating with the good polarization.

Another possibility is to heat at harmonics. For example, one can heat a pure D plasma at the second harmonic $\omega=2 \omega_{c D}$ with the same polarization as above, or at the third harmonic $\omega=3 \omega_{c D}$ with

$$
\frac{E_{L}}{E_{R}} \approx \frac{3 \omega_{c D}-\omega_{c D}}{3 \omega_{c D}+\omega_{c D}}=\frac{1}{2} .
$$

When working with a mixture of gases, for example, majority $\mathrm{D}$ with some amount of $\mathrm{H}$ [a mixture denoted $\mathrm{D}-(\mathrm{H})]$, the FW usually does not propagate throughout the plasma, except for a very small concentration of the minority. Instead, the plasma cross section is separated into two propagating zones by the confluence layer (Fig. 1). On the LFS of this layer (right dashed line in Fig. 1), the wave is evanescent, which means that waves launched from its right will be reflected by this layer and bounce inside a kind of immaterial cavity, delimited by the confluence layer cutoff and the outer cutoff, and will transfer resonantly their energy to ions at each crossing of the $\left(f=f_{c H}\right)$ cyclotron layer. On the contrary, waves launched from the HFS (rays coming from the left in Fig. 1), rather than being reflected, will be mode converted when they reach the confluent zone. This means that from the originally long-wavelength electromagnetic type of waves they were as the FW, they will be transformed into short-wavelength electrostatic waves. Depending on the path of the ray, the converted wave will be either an ion Bernstein wave (rays closer to the equatorial plane in Fig. 1), propagating backward to the HFS, or an ion cyclotron wave (top and bottom rays to the left of the confluence layer in Fig. 1) that will tend to continue propagating toward the $f=f_{c H}$ cyclotron layer. Most of the energy of these short-wavelength modes will be damped on electrons by Landau damping. A heating scenario where most of the waves are converted to shortwavelength modes is called a mode-conversion scenario and, in TEXTOR, corresponds to dominant electron heating. The mode-conversion process has been analyzed in detail by Perkins. ${ }^{5}$

\section{THE TEXTOR ION CYCLOTRON HEATING SYSTEM}

The TEXTOR ion cyclotron frequency heating system was put into operation in 1984. It consists of two independent power lines, initially designed ${ }^{6,7}$ to generate and launch in the machine $1.5 \mathrm{MW}$ of radio-frequency (rf) power each, during $3 \mathrm{~s}$ in the frequency range 25 to $29 \mathrm{MHz}$. Each power 1ine included a $\lambda / 4$ stainless steel half-turn antenna of the shielded strip-line type (Fig. 2) placed along the machine's liner. The central conductor could be replaced by a twice-narrower conductor. Each antenna is coupled to the pressurized transmission line

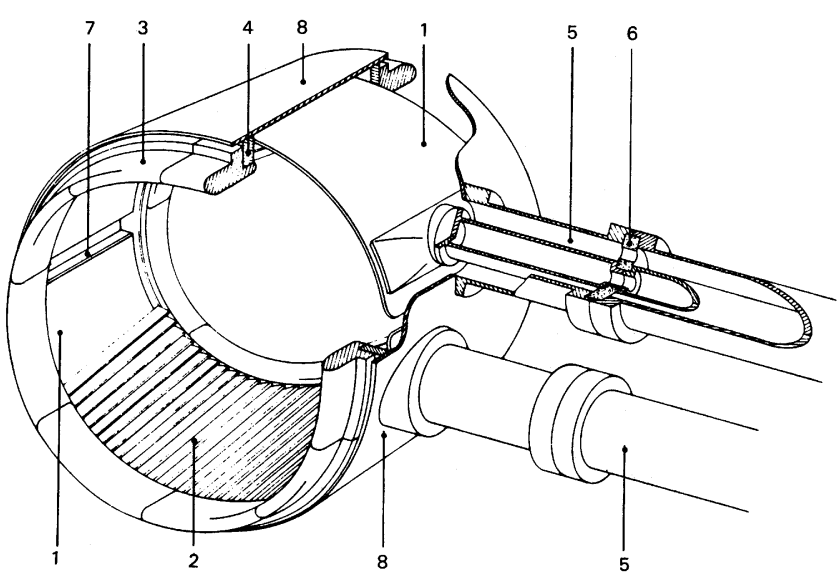

Fig. 2. Perspective view of the first ICRH antennas: $1=$ central conductor, $2=$ electrostatic screen, $3=$ guard limiter, $4=$ support flange, $5=$ coaxial line, $6=$ line and antenna support, $7=$ short-circuit, and $8=$ TEXTOR liner. 
by means of an interface including a vacuum coaxial line; a ceramic feedthrough; and a fixed stub, which performs a first impedance matching.

At the beginning of 1987, a new phase in the TEXTOR program was initiated with the installation of the Advanced Limiter Test II (ALT-II) pumped toroidal belt limiter followed in the spring of 1988 by the installation of neutral beam heating at a level of $2.6 \mathrm{MW}$. The ICRH system was also upgraded in 1987-1988. The modification of the ICRH system involved three steps:

1. In 1987 four new movable antennas replaced the two previously installed ones. Contrary to the previous antennas that were launching power predominantly from the HFS (Sec. III), the new antennas launch power from the LFS, leading to substantially different physics scenarios (Sec. IV and subsequent sections). New interfaces and additional transmission lines were installed at the same time.

2. Two new fully regulated power supplies were acquired allowing higher-power ratings, better operation under mismatched conditions, and longer (3-s) pulses.

3. More powerful tetrodes were installed in the generators, and auxiliary hardware was modified to bring the generator's output power to $2 \mathrm{MW}$. The generator frequency band was increased to 25 to $38 \mathrm{MHz}$ to operate at the new upgraded value of the toroidal magnetic field $(2.6 \mathrm{~T})$ in the most efficient $\mathrm{D}-(\mathrm{H})$ minority scenario.

A detailed technical description of the TEXTOR system is given in Ref. 8 .

A new feedback-controlled real-time matching system, based on variable capacitors, was designed and installed in 1992 (Ref. 9). In 1994, TEXTOR was upgraded to allow longer pulses, and the pulse length of the ICRH system was increased to $10 \mathrm{~s}$.

In the course of time, several different versions of the four-strap antenna system were installed, allowing the investigation of different heating physics aspects. This will be described below.

In 1996, a new feedback control system was put into operation, which keeps constant the total energy content of the plasma by regulating the rf power. Combined with the feedback control of the radiated power by the impurity influx, it allowed the performing of stationary long pulses with high confinement in the radiatively improved (RI) regime (see Sec. V.B) (Ref. 10).

Analysis of present-day ICRF amplifiers and measurements carried out on TEXTOR's ICRF system has revealed spurious generation of power at the harmonics of the operating frequency. Filters have been designed that eliminate the most prominent of these unwanted components, i.e., the second-harmonic one. In 2001, these filters were installed on TEXTOR and successfully tested. $^{11}$

\section{EARLY EXPERIMENTS AND MODE-CONVERSION PHYSICS}

The first antenna system installed on TEXTOR is shown in Fig. 2. It consisted of two half-turn antennas, one at the top half of the poloidal cross section and the other at the bottom. The central conductor that carries the rf current is visible on the cutaway. The current flows in the poloidal direction and has approximately a quarterwavelength $(\lambda / 4)$ cosine shape with the maximum at the short circuit (HFS) and the zero in the vicinity of the feeding point (connection to the coaxial feeder). A Faraday shield (FS) that polarizes the electric field in the direction perpendicular to the total magnetic field protects the strap. In the antenna cross section, the main rf magnetic field component is toroidal. The Poynting vector (power-flux vector) near the antenna surface points radially toward the plasma center. This is why the rays representing waves radiated by the antenna start in the radial inward direction on Fig. 1, which gives a geometricoptics representation of the field radiated by the antenna. Because the maximum of the rf current is at the HFS and the power flux at the antenna is proportional to its square, most of the power is launched from the HFS. For this reason, most rays start from the HFS in Fig. 1, and in consequence, the heating scenario with such an antenna is mostly mode conversion. Dominant ion cyclotron heating can be obtained only in a minority scenario with a very small concentration of the minority.

In the first experiments in a full-metal machine (INCONEL ${ }^{\circledR}$ liner, stainless steel antennas), the ohmic discharges in $\mathrm{D}$ were characterized by a rather large $Z_{\text {eff }}$ $(>3)$. While a modest electron heating was observed, the edge plasma and the scrape-off layer (SOL) were profoundly perturbed during the rf pulses, bringing the plasma to a predisruptive state characterized by exponential increases of impurity lines and of the fluctuation level. This limited rf operation to low-energy pulses, e.g., $350 \mathrm{~kW}$ for $10 \mathrm{~ms}$ and $55 \mathrm{~kW}$ over $1.2 \mathrm{~s}$ (Ref. 12).

High rf power operation could be achieved after wall conditioning by carbonization. A strong modification of the plasma-surface interaction was obtained by the deposition of carbon layers, resulting in a strong reduction of the radiation from heavy impurities and of the level of oxygen. This allowed reaching the megawatt-power and megajoule-energy ranges. The global energy confinement time was observed to degrade with additional $\mathrm{rf}$ heating but found to scale with the power and the plasma parameters like in the case of neutral beam heating experiments, ${ }^{13,14}$ indicating an equivalent heating efficiency for the two heating methods. This confinement degradation can be understood by assigning to the additional heating power a specific incremental confinement time $\tau_{\text {aux }}$ (ratio of the increase in energy by the additional power) distinct from the global energy confinement time and by assuming that only a fraction $\alpha$ of the rf power is coupled to the plasma bulk (as opposed to a part that 
could be dissipated near the edge and would not contribute to the efficient heating of the plasma). Fitting the two parameters $\alpha$ and $\tau_{a u x}$ to the experimental energy response of the plasma to $\mathrm{rf}$ transients allows characterizing the efficiency of power coupling to the plasma and the amount of confinement degradation. Comparison with the theoretical predictions using ray-tracing (as in Fig. 1) showed good agreement. Going to very low concentrations of the minority (below $\sim 1 \%$ ) allowed first exploration of ion heating, with second-harmonic absorption beginning to dominate electron heating.

The scenario with ${ }^{3} \mathrm{He}$ minority in $\mathrm{D}$ was also investigated. It has much weaker absorption than the D- $(\mathrm{H})$ scenario. The experimental results nevertheless indicate fairly high absorption efficiency in the lowest concentration range. This efficiency falls dramatically at higher concentrations, and all heating completely vanishes above $8 \%$ of ${ }^{3} \mathrm{He}$.

\section{LFS LAUNCH AND PREDOMINANT ION HEATING}

In 1987 the new antenna system shown in Fig. 3 was mounted on TEXTOR. The antenna pair shown in Fig. 3 was connected to one of the generators, and a similar pair positioned at the toroidally opposite location in TEXTOR was connected to the second generator. Each pair could be operated in phase or in phase opposition. Connecting the two generators to one pair allowed arbitrary phasing.

\section{IV.A. Coupling}

Figure 4 shows a comparison between the predictions of the coupling model ${ }^{15}$ and the experimental measurements on TEXTOR of the antenna specific resistance $R$ versus $\mathrm{Ne}_{40}$, the line-averaged density at $r=40 \mathrm{~cm}$ (the plasma radius is $r=46 \mathrm{~cm}$ ). Profiles have been chosen according to experimental interferometric measurements. The comparison is made for the "OLD" antenna (Fig. 2), and the new one (Fig. 3) with current excitation in phase (zero-phase) or in phase opposition ( $\pi$ phase). For the OLD antenna, theory is in agreement with the low-power experimental points. High-power coupling resistances are approximately twice lower, indicating that some important profile modifications were taking place in front of the initial antennas. The agreement is very good for the new antennas in $\pi$ phasing, but for zero phasing, the experimental resistance shows an edge density dependence that is not predicted by theory. It might be due to density profile modifications induced by the rf itself. The excitation of FW global eigenmodes was investigated both experimentally and theoretically. ${ }^{16}$ The behavior of the following signals was studied: resistance and inductance of the antennas, $\mathrm{rf}$ magnetic and electric field components picked up by probes at the plasma edge,

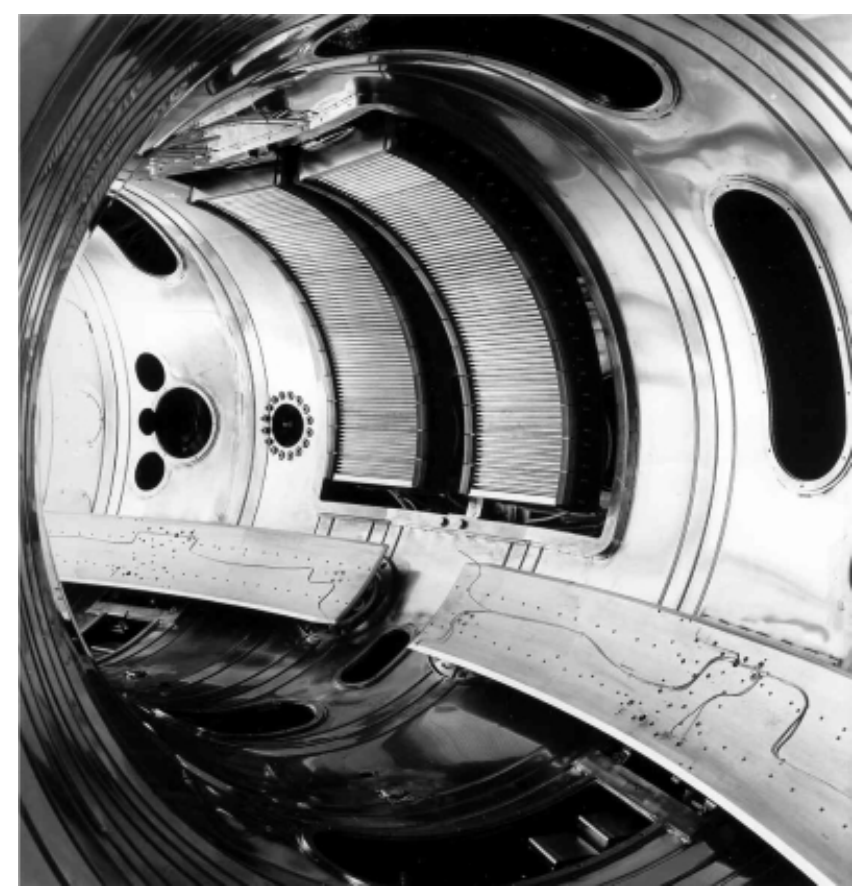

Fig. 3. One pair of the antennas compatible with the ALT toroidal belt limiter mounted in 1987.

and power coupled to an FW antenna used as a receiver. It was shown that all the major features of these signals linked to the existence of eigenmodes could be explained by the theoretical models.

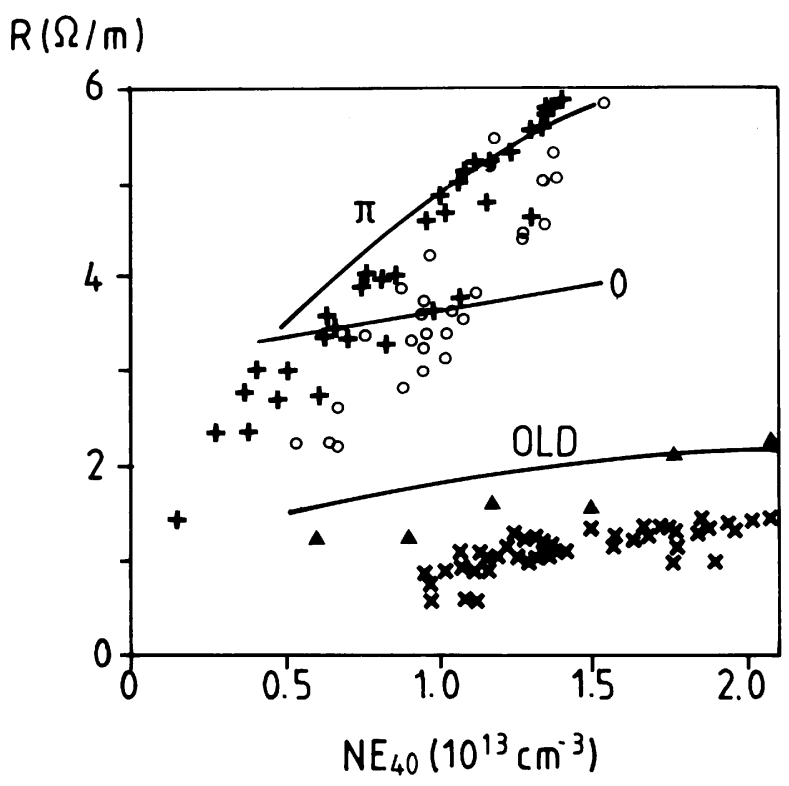

Fig. 4. Comparison between experimental and theoretical specific resistance of the TEXTOR antennas: $(x)$ OLD antenna, high power $(>200 \mathrm{~kW}) ;(\boldsymbol{\Delta})$ OLD antenna, low power $(\approx 40 \mathrm{~kW}) ;(+)$ new antenna $\pi$ phasing; $(\mathrm{O})$ new antenna 0 phasing. 


\section{IV.B. Effect of Wall Conditioning}

Following the initial observation of the importance of wall conditioning for good ICRH operation, the plasma-wall interaction during ICRH was further studied. A reduction of this interaction can be obtained by an appropriate choice of the wall conditions:

1. A factor 5 reduction of the density increase induced by ICRH has been obtained by going from carbonized wall, zero phasing operation to boronized wall with $\pi$ antenna phasing.

2. The values of the total and incremental power radiated fractions are strongly dependent on machine wall condition. They are strikingly lower in the case of boronized wall.

3. Because of the much easier density control achieved with a boronized wall, it is possible to raise the rf power near the maximum available value in the full density range.

The reduction of plasma-wall interaction achieved with wall conditioning allowed routine long pulse ( $>1-\mathrm{s}) \mathrm{ICRH}$ operation on TEXTOR near the maximum power available ( 2 to $2.8 \mathrm{MW}$ ) at that time.

\section{IV.C. Phasing}

The interaction between the plasma and the wall (liner and limiters) is modified by switching from $\pi$ to zero antenna phasing, dependent wall conditions. In the Ref. 17 study, a significantly lower oxygen radiation was observed with $\pi$ phasing, whereas the carbon radiation showed an opposite, yet much weaker, tendency. As a result the total radiated power fraction and the incremental one (due to $\mathrm{rf}$ only) were found to be lower for $\pi$ phasing. The density increase due to ICRH is somewhat lower for $\pi$ phasing. The different impact on recycling of the zero- and $\pi$-phase configurations is seen in a reduction of limiter interaction in zero phasing together with increased interaction with the liner, possibly leading to plasma detachment. With the $\pi$ phasing no sign of detachment was observed.

\section{IV.D. Confinement}

Notwithstanding the change from HFS to LFS antennas, the heating efficiencies and the confinement properties were found to be in line with the previous results for the OLD antenna configuration. They are roughly independent of the phasing configuration or of the different wall conditions used. As expected from the LFS location of these new antennas, an energetic hydrogen tail is observed due to the predominantly hydrogen minority heating scenario.

\section{IV.E. Edge Physics}

Edge density measurements were made with Li beams having a small connection length to one of the antennas. A significant modification of the SOL was observed (Fig. 5) during the rf pulse. This perturbation is probably not uniform as these modifications were not measured with other diagnostics located at different connection lengths. ${ }^{17}$

Measurements performed with electric and magnetic rf probes in the SOL of TEXTOR during ICRF operation revealed the presence of large-amplitude harmonics of the rf generator frequency, due to parametric decay instabilities. The first type was identified as decay into an ion Bernstein wave and an ion cyclotron quasi-mode; the second was identified as decay into an ion Bernstein wave and a low-frequency electron quasi-mode. Signals at half-harmonics of the generator frequency were also seen and interpreted as parametric FW pump decay into two ion Bernstein waves in the interior of the plasma. ${ }^{18}$

Measurements of the direct-current (dc) flowing between a Faraday-shielded ICRF antenna and the wall (liner) were performed on the ICRF-heated TEXTOR plasma. It was observed that the dc, corresponding to electrons drawn by the antenna, increased by a factor of 8 , reaching a value of 80 A for $200-\mathrm{kW}$ rf power. This increased dc current was attributed to plasma sheath effects at the antenna. ${ }^{19}$

Radial profiles of the de radial and poloidal fields in the SOL were measured in ohmic- and ICRF-heated discharges by means of a fast scanning double probe. It is found that ICRF heating significantly changes both field components. Poloidal fields as high as $10 \mathrm{~V} / \mathrm{cm}$ have been observed at $1 \mathrm{MW}$ of ICRF power. ${ }^{20,21}$

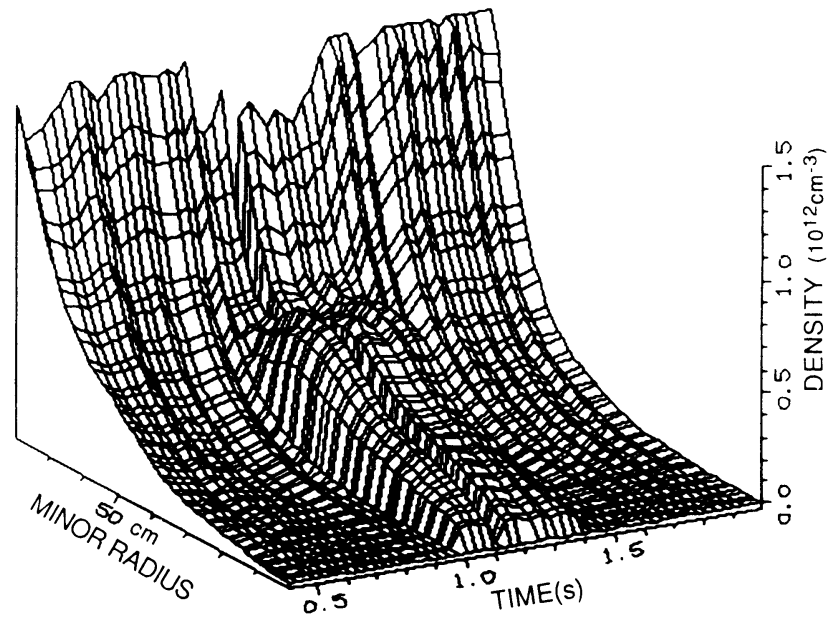

Fig. 5. Three-dimensional plot of the edge density profile in the outboard side of the equatorial plane versus of time, from thermal Li beam spectroscopy. ${ }^{17}$ 


\section{COMBINED NBI-ICRF HEATING}

\section{V.A. Confinement}

At the time the two neutral beam injectors became available on TEXTOR, heating with the different combinations of systems was performed. The two beams are injected tangentially into the plasma, one in the direction of the plasma current [neutral beam injection (NBI)-co] and, the other in the opposite direction (NBI-counter). Early experiments with NBI-co and ICRH have essentially shown that plasma energy increases proportionally to the combined total power $\left(P_{a u x}=P_{\mathrm{NBI}_{c o}}+P_{\mathrm{rf}}\right)$. Later, larger power could be injected using combinations of the three different heating systems. With NBI, a large energy anisotropy was generally observed, revealing a tail part in the energy content. A large enhancement (factor above 2) with respect to the Kaye-Goldston scaling was found with both combinations NBI-co alone, NBI-co + ICRH or NBI-co + NBI-counter, with a maximum enhancement of 1.5 for the thermal contribution only. With the three heating methods combined, a value of the poloidal beta $\beta_{p}=1.5$ was reached corresponding to a toroidal $\beta_{T}=0.65 \%$, i.e., $70 \%$ of the Troyon limit.

Complete sawtooth stabilization could be obtained by the hot ion tail produced by NBI-co. The density domain in which stabilization occurs was significantly extended by the addition of ICRH (Ref. 22).

\section{V.B. Radiatively Improved Confinement Mode}

With the additional injection of impurities (impurity seeding mainly with neon and argon), the above improved confinement with NBI-co or combined heating was further increased. Contrary to expectations, impurity injection was not accompanied by a substantial increase in $Z_{\text {eff }}$, even though leading to substantial increase of the radiated power in the boundary. Impurity seeding finally allowed pushing the density near to and even above the Greenwald limit. (RI)-mode physics is discussed in more detail in Ref. 23.

\section{V.C. Current Drive and Rotation}

During combined heating experiments of NBI-co + ICRH, a large increase of beam-driven current could be produced in TEXTOR (up to $70 \%$ increase depending on the mix of NBI and ICRH power). This, together with the enhancement of the bootstrap current, resulted in a large volt-second saving, allowing substantial increase of the shot duration (e.g., by a factor of 5 at $I_{p}=300 \mathrm{kA}$ ). A large part of the synergistic increase of the beam-driven current is explained by the rise of the electron temperature produced by ICRH, which leads to an increase of the beam slowing-down time. A smaller contribution to the effect is due to direct coupling of the rf power to the beam ( $\sim 20 \%$ of the rf power) and to the decrease of the toroidal rotation velocity induced by the $\mathrm{rf}$ (Ref. 24).

\section{V.D. Impurities}

Figure 6 shows an overall view of bolometric measurements during auxiliary heating for discharges of equal density. The dependence of the radiated power on the auxiliary power does not show any deviation from linearity for the different heating methods or their combinations, excluding any rf-specific impurity production. The analysis of the Fe XXI line confirms the bolometric result. ${ }^{25}$

\section{FAST PARTICLE EFFECTS}

\section{VI.A. Third-Harmonic Heating}

At a frequency of $38 \mathrm{MHz}$ and a magnetic field below $1.8 \mathrm{~T}$, on TEXTOR, the second-harmonic D (and hence fundamental $\mathrm{H}$ ) is outside the plasma. Then, the thirdharmonic deuterium cyclotron layer, located near the plasma center, is the only cyclotron resonance in the plasma. Because the efficiency of damping of the rf waves by the thermal plasma decreases strongly with the harmonic number, in ohmic plasmas, this resonance gives only very weak absorption. The only remaining absorption mechanism is electron damping, which is also very weak. In this weakly absorbing plasma, small amounts of rf power cause large density excursions, and attempts to force more power into the plasma lead to disruption. To increase the electron temperature and thus the electron damping, NBI heating (D injection) was added to the discharge. This allowed the coupling of reasonable amounts of rf power but led to results that contradicted the assumption of a pure electron heating scheme. As Fig. 7 shows, there is nearly no electron heating while the neutron production (mainly due to beam-target reactions) is nearly proportional to the total additional power. This is explained by the heating of the beam ions by the

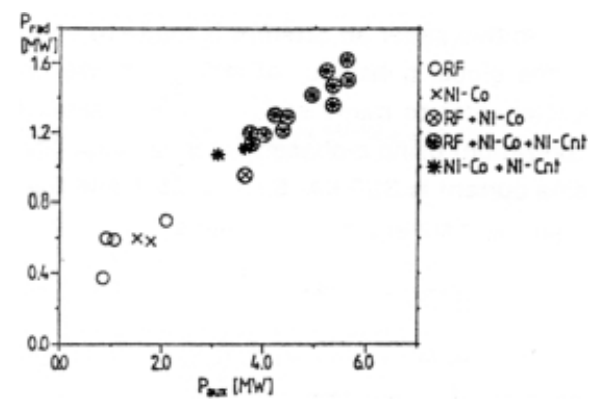

Fig. 6. Radiated power (from bolometry) versus auxiliary heating power for ICRH, NBI-co, and NBI-counter, and their combinations. 


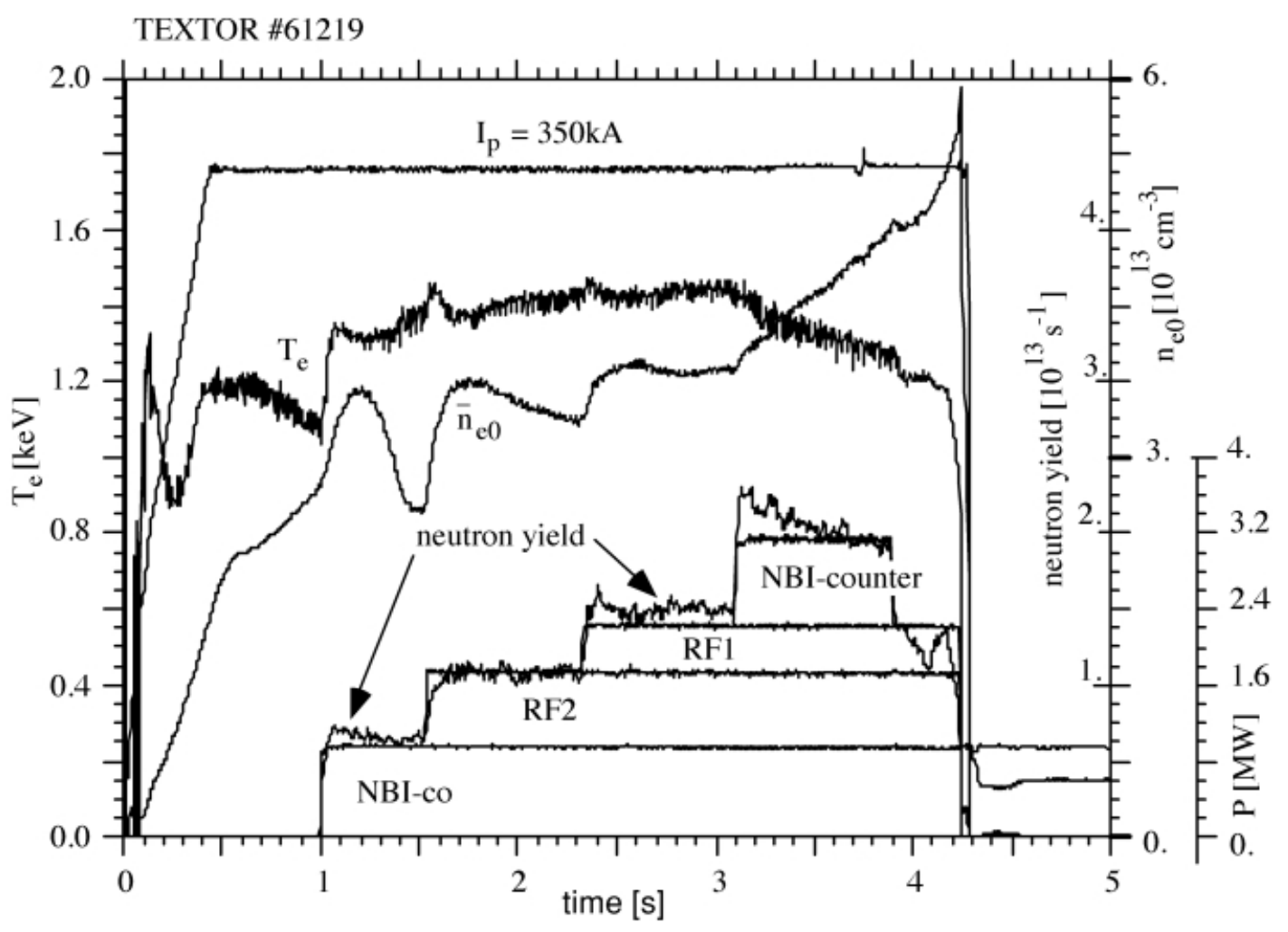

Fig. 7. Example of a TEXTOR shot with combined NBI and third-harmonic rf heating.

rf, which has become the dominant rf absorption mechanism. The D-beam ions absorb rf power much more efficiently than the thermal D because they are so much faster, as theory shows.

In this scenario, the experimental energy increase obtained from the successive power steps is in some cases two to three times larger than usual, a behavior indicating that there must be large changes in transport induced by the rf. Another characteristic of the energy behavior in third-harmonic heating is a much stronger dependence on density than the standard ITER L89-P scaling law. These features are reminiscent of the transitions to improved confinement accompanied by strong density dependence that one also gets on TEXTOR using edge radiative cooling, although in the present shots, no such cooling was used. ${ }^{26}$

\section{VI.B. Transport Control of ${ }^{3} \mathrm{He}$}

Measurements ${ }^{27}$ were performed to test a model prediction that states that the off-axis coupling of ioncyclotron waves to energetic helium ions can generate either an inward or an outward drift of these particles. Ion cyclotron waves with a power of up to $1 \mathrm{MW}$ were coupled to energetic particles (from NBI) at the HFS, LFS, or in the center by varying the toroidal magnetic field. When changing the heating power or the resonance location, the variation of the concentration of the energetic helium in the plasma was seen to agree with the model predictions.

\section{TEST OF AN ELECTROSTATIC ANTENNA}

An electrostatic type of antenna was tried on TEXTOR allowing a maximum of $340 \mathrm{~kW}$ to be coupled to the plasma during long pulses $(0.5 \mathrm{~s})$. Possibly because of mechanical problems (the central conductor dielectric supports were found to be broken after the experiments, leaving the top part of the central conductor hanging much more closely to the plasma edge than planned), the voltage standoff was found to be only half that of the regular FW antenna. The coupling capability itself, in terms of power coupled for a given maximum voltage on the antenna, was also a factor of 2 lower than that of the FW antenna. The coupling resistance did not show the characteristics of an ion Bernstein wave coupler, but altogether, theoretical investigations indicate that this antenna is mainly a FW launcher. The edge perturbations caused by this antenna were almost negligible while bulk density increase was observed, accompanied by a proportional energy increase. ${ }^{28}$ 


\section{WORKING WITH UNSHIELDED ANTENNAS}

\section{VIII.A. Operation}

The introduction of an FS in ICRH antennas was essentially of empirical origin; it was a recipe to improve the heating efficiency and avoid voltage breakdown, arcing, impurity generation, etc. Different distinct functions have been attributed to the FS. It acts as a polarizer, coupling fields that match the polarization of the FW and avoids coupling to the slow wave. It isolates the antenna central conductor from the edge plasma, which could otherwise prevent effective coupling, short-circuit the conductor, or cause other negative side effects such as arcing and impurity generation. A third function is technical: The screen is a slow wave structure that shortens the current wavelength along the strap.

On the basis of a revisited analysis of the role of the FS (Ref. 29) predicting no harmful effects for TEXTOR antennas, the screen was removed from a pair of antennas - with feeder area covered, as shown in Fig. 8and power was coupled to the plasma with success. No problems (regarding impurity, arcing, coupling, etc.) were encountered in operating the screenless antenna not only in $\pi$ phasing but also in zero phasing, even at high $\mathrm{rf}$ power $\left(P_{\mathrm{rf}}=1.6 \mathrm{MW}\right)$. Basically, in $\pi$ phasing, there was no difference between shielded and unshielded antennas, except a higher Fe XXI signal in some cases. For zero phasing, a comparison was made under boronized wall conditions, with and without NBI. As reported in Sec. IV.C, operation in $\pi$ phasing significantly reduces the interaction with the wall, as compared to zero-phase operation. However, no difference between zero phasing and $\pi$ phasing was observed in these experiments with unshielded antennas, possibly because of a fresh boronization masking the effect of specific mechanisms of rf- edge interaction. As well, no systematic difference could be found in the plasma energy content.

In the analysis of the unshielded antenna operation, it was thought that the protection of the feeder area (see Fig. 8) could play an important role in avoiding adverse effects because it masks a region of high fields with ill-defined polarization to the plasma. To check this point, the protection blades were removed from the unshielded antenna, and a comparison was made with a shielded antenna (with protection blades). These experiments (ICRH alone) showed striking differences with respect to the earlier situation. The unshielded antenna now gave significantly smaller energy increments than the shielded one. The differences were more marked on the diamagnetic energy signal at large $P_{\mathrm{rf}}$ (maximum 1.4 MW), under which conditions the energy increment for the unshielded antenna could be only half of that of the shielded one. The unshielded antenna tended to cause disruption earlier than the other. However, it is remarkable that when the $\mathrm{rf}$ is operated simultaneously with the neutral beams, all these differences vanish, and the two antennas appear to behave identically. ${ }^{30}$

Since these experiments, all types of antennas on TEXTOR have been constantly equipped with feeder protection and operated without an FS and without any problem. The maximum power could be coupled (meaning that the operation is not voltage limited) at the highest densities under RI-mode conditions.

\section{VIII.B. Ponderomotive Forces}

Langmuir probe measurements have been made inside an ICRF antenna without a Faraday screen. It was found that upon rf switch-on, the low-density plasma between the antenna side protection limiters (see Fig. 8) is promptly pushed out of this region, even at very low $\mathrm{rf}$

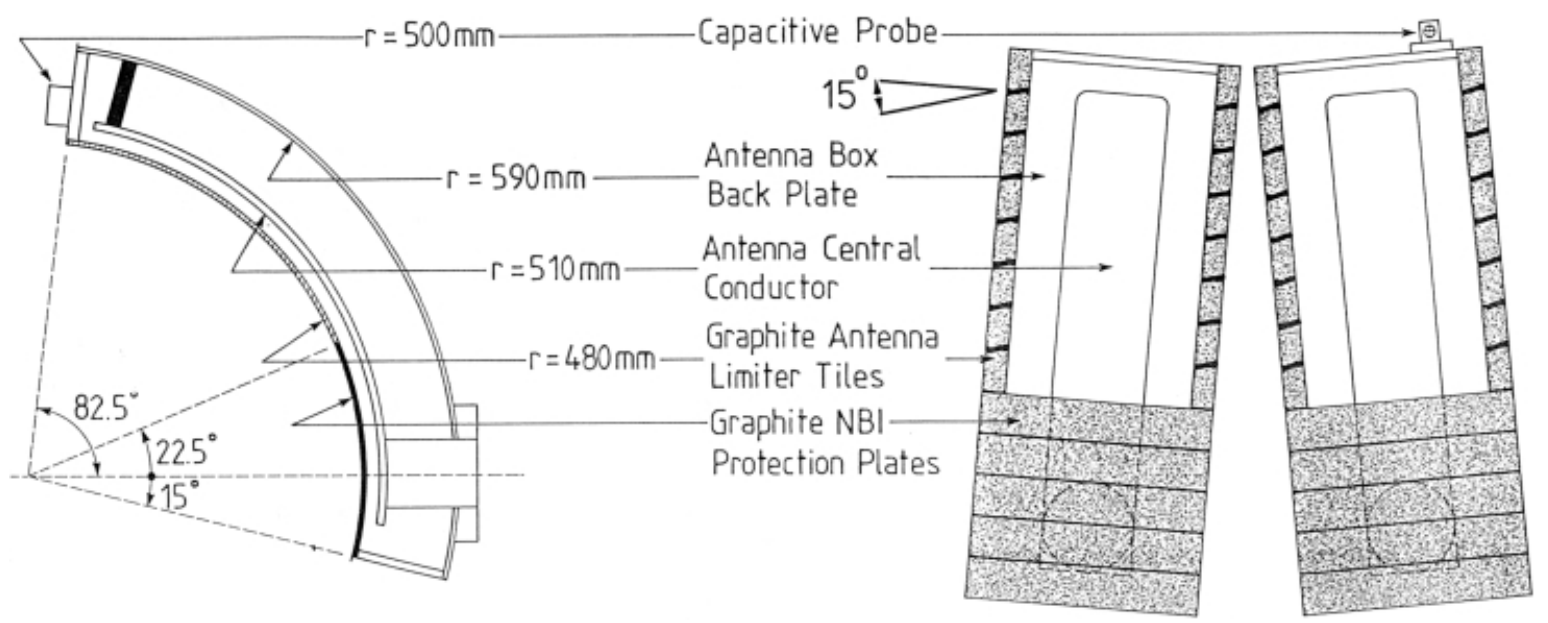

Fig. 8. Layout of the unshielded ICRH antenna. The feeding point at the bottom of the straps is hidden to the plasma by graphite plates. The side protection limiters of the antenna, made out of graphite tiles, are also shown. 
power (order $1 \mathrm{~kW}$ ). This means that any plasma present inside the antenna box before the onset of the rf is quickly blown away by the rf itself. An order of magnitude estimate indicates that this effect could be attributed to the ponderomotive force linked to the large parallel electric fields close to the antenna. Because of this effect, the screenless antenna can be operated at much higher densities than would be expected from simply taking into account cross-field diffusion in the absence of $\mathrm{rf}$ to estimate the density at the strap level. This is confirmed by the fact that on TEXTOR, the screenless antenna still works properly at the largest available density. ${ }^{31}$

\section{TESTING NEW ANTENNA TYPES}

\section{IX.A. Low Electric Field Antenna}

In 2001, on one of the two antenna pairs of TEXTOR, the thin radiating strap was replaced by a set of three cylindrical bars (see Fig. 9). This allows decreasing significantly the maximum electric field, on the sides of the strap, for a given radiated power. Two-dimensional

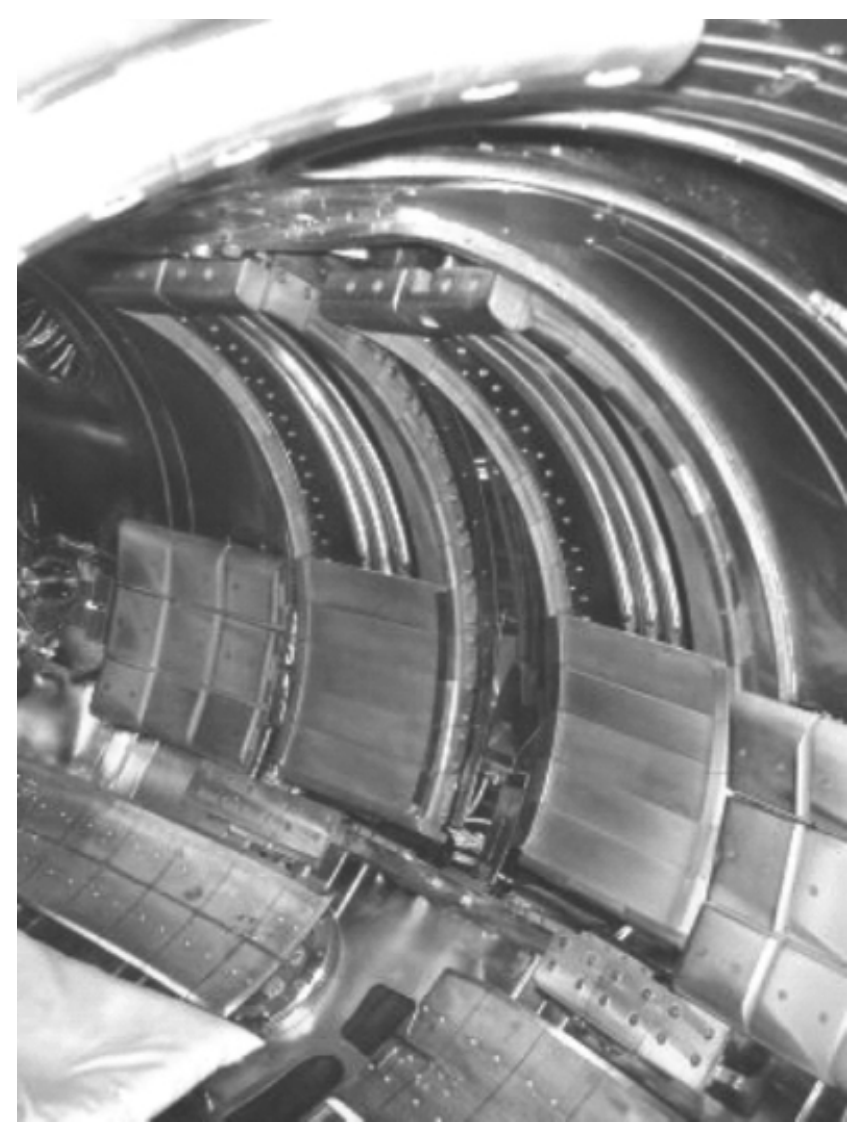

Fig. 9. Low electric field antenna. The picture shows the central conductor made of three tubes instead of one strap. Like all others, this antenna is operated without an FS. electrostatic modeling shows that for the same voltage $V_{A}$ applied to the hot conductor, the maximum electric field is reduced by a factor 1.6. However, as the rf current density is larger at the inner side of the hot conductor, there is a loss of coupling to the plasma and the low electric field antenna resistance is $\sim 30 \%$ lower. The low electric field antenna was routinely operated with high reliability up to the full installed power (2 MW, with up to $95 \%$ radiated in the plasma, for both antennas). If the power limitation would be due to arcing between the strap and the antenna box one could expect an improvement in the power-handling capability of the low electricfield antenna by a factor 2.3 (Ref. 32).

\section{IX.B. Load Insensitive Antenna}

Because of the rearrangement of the diagnostic positions resulting from the dynamic ergodic divertor ${ }^{33}$ installation on TEXTOR, a new antenna system had to be designed to be compatible with the inlet of the diagnostic beam of TEXTOR between its two radiating strips. The design was made such as to be able to test the "conjugated T"mode of operation that is foreseen for the new Joint European Torus (JET) ITER-like antenna. ${ }^{34}$ This mode would be fairly insensitive to the variations of the antenna loading resistance and could help solve the problem of generator tripping occurring in the ICRH heating of Elmy H-mode plasmas. Side and top views of the new antenna pair are shown, respectively, in Fig. 10. The two identical radiating straps are fed by their feeding lines at a tap B. They are short-circuited at one side and connected at the other side to a vacuum variable capacitor (grounded in E) by means of a section C-D of coaxial line. The two feeding lines are connected to the line coming from the generator, of which the length can be adjusted by means of line stretchers. This new antenna was mounted on TEXTOR in spring 2004.

\section{$X$. PLASMA PRODUCTION WITH rf}

Plasma was produced for the first time with ICRH power only in a tokamak (TEXTOR) in 1994 (Ref. 35). The RF discharge was performed in the presence of a (toroidal) magnetic field and in the absence of plasma current.

\section{X.A. ICRF Wall Conditioning}

One motivation for plasma production by $\mathrm{rf}$ is that in future reactor-scale superconducting fusion devices, such as ITER, the presence of a permanent high magnetic field will prevent the use of glow discharge conditioning (G-DC) between shots, which is presently the preferred method for wall conditioning. The need of controlled and reproducible plasma startup and tritium removal, e.g., from the codeposited carbon layers, will require applying 


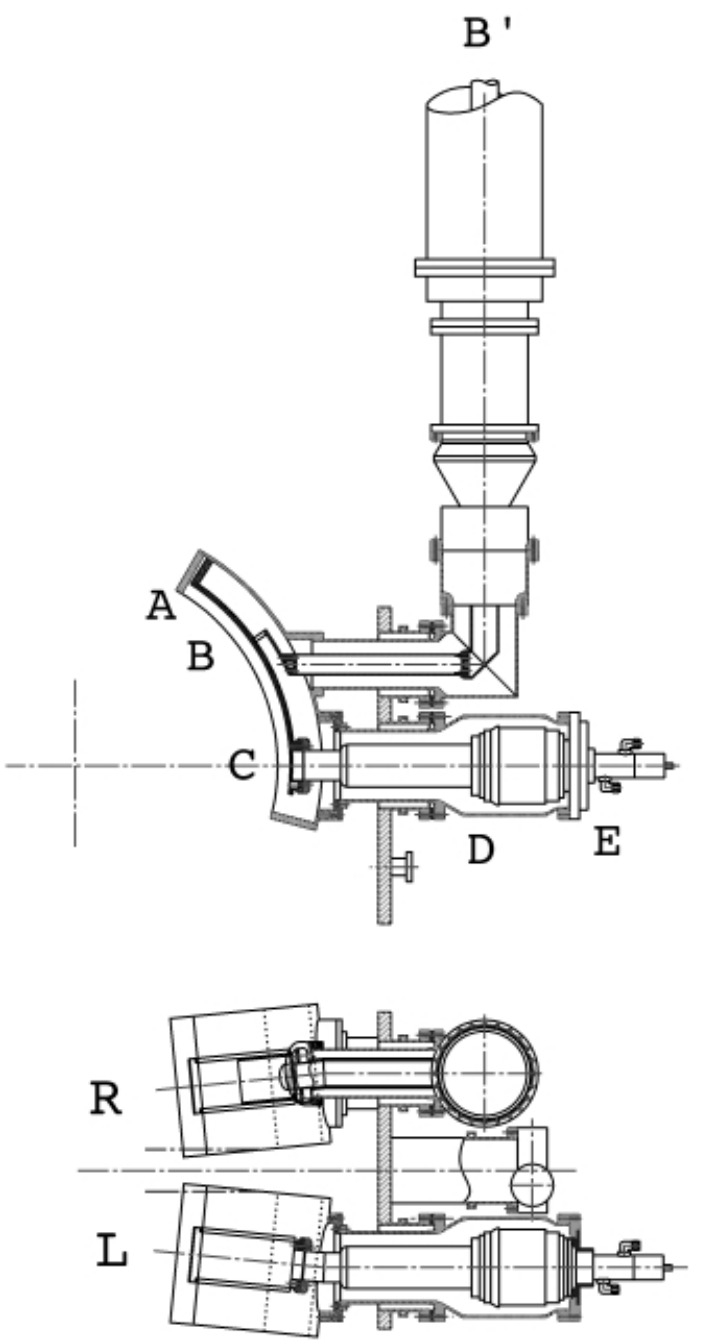

Fig. 10. Side and top views of the tunable antenna pair. The top view shows on the $\mathrm{R}$ side the upper part and on the $\mathrm{L}$ side the lower part.

an alternative wall-conditioning procedure. Discharge conditioning with waves in the electron cyclotron range of frequencies (ECRF) and ICRF (ECRF-DC and ICRF$\mathrm{DC}$, respectively) is fully compatible with the presence of a magnetic field.

ICRF-DC has been developed in TEXTOR using the existing ICRF system without any change in hardware. It was performed in the presence of the second-harmonic He or D cyclotron resonance (Fig. 11). The ICRF-DC wall conditioning efficiency has been compared with that of ECRF-DC (Ref. 36). The hydrogen removal rate has been found to be about 20 times higher in ICRF-DC discharges $\left(\omega \approx 2 \omega_{c i}\right)$ than in ECRF-DC discharges $(\omega \approx$ $\left.2 \omega_{c e}\right)$ produced by a focused microwave beam. The most probable reason for this is the more homogeneous plasma density profile $\left(n_{\mathrm{e} 0} \approx(2\right.$ to 4$\left.) \times 10^{17} \mathrm{~m}^{-3}\right)$ and the generation of high-energy ions (detected in the range $E_{\mathrm{i}} \approx$

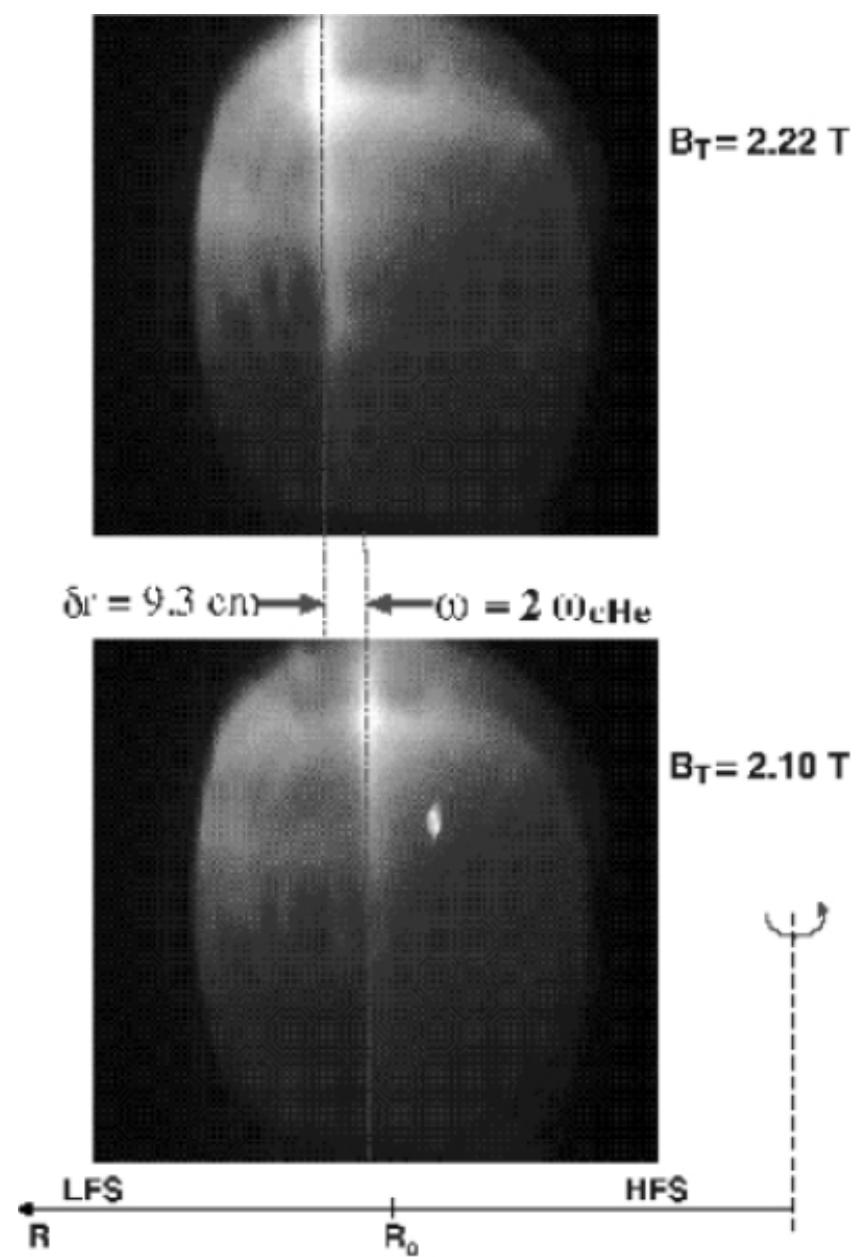

Fig. 11. Charge-coupled-device view of ICRF plasmas with vertical radiating layer associated with position of the $\omega=2 \omega_{c \mathrm{He}}=\omega_{c \mathrm{H}}$ cyclotron resonance.

4.4 to $55 \mathrm{keV}$ ) with ICRF-DC (Ref. 37). However, the combination of ECRF and ICRF power seems promising for decreasing the $\mathrm{rf}$ voltage at the ICRF antenna and improving the rf power coupling (up to $36 \%$ ).

\section{X.B. ICRF-Assisted Low Loop Voltage Tokamak Start-Up}

A second motivation is to allow reproducible lowvoltage start-up of the plasma current. For the present ITER start-up scenario, the inductive electric field is limited to $E \approx 0.3 \mathrm{~V} / \mathrm{m}$ in the breakdown region to prevent a quench in the superconducting coils. To perform start-up reliably at such low electric field, noninductive preionization, target plasma production, and preheating are needed

ICRF-assisted low loop voltage start-up has been successfully tested on TEXTOR (Ref. 38). The two pairs of the ICRF double-loop antennas without an FS, driven in $\pi$ phase, have been used in the standard $2 \omega_{c i}$ scenario 
for the preionization and target plasma preheating. ICRFassisted start-up was achieved at the central inductive electric field $E_{0} \approx 0.32 \mathrm{~V} / \mathrm{m}$, which meets ITER requirements. Without assistance, $E_{0}$ is $\sim 0.45 \mathrm{~V} / \mathrm{m}$. ICRFassisted start-up has been found to be more prompt and robust than a nonassisted one and has resulted in a significantly (about four times) broader pressure range for current initiation, with $\sim 22 \%$ higher current ramp-up rates.

\section{REFERENCES}

1. T. H. STIX, The Theory of Plasma Waves, McGraw-Hill (1962).

2. T. H. STIX, Waves in Plasmas, American Institute of Physics (1992).

3. R. KOCH, Trans. Sci. Fusion Technol, 41, 183 (2002).

4. A. NICOLAI, P. BOERNER, R. KOCH, and D. VAN EESTER, Proc.14th European Conf. Controlled Fusion and Plasma Physics, Madrid, Spain, Europhysics Conf. Abstracts 11D, 1105 (1987).

5. F. W. PERKINS, Nucl. Fusion, 17, 1197 (1977).

6. V. P. BHATNAGAR et al., in Proc. 12th Symp. Fusion Technology (SOFT), Jülich, Germany, Pergamon Press, Vol. 2, p. 1275 (1982).

7. J. M. NOTERDAEME, R. WEYNANTS, and A. MESSIAEN, "Engineering Problems of Fusion Research," in Proc. 9th Conf., Chicago, Illinois, 1981, Vol 1, p. 831, Institute of Electrical and Electronics Engineers (1981).

8. G. VAN OOST et al., Fusion Technol. 12, 449 (1987).

9. F. DURODIÉ, Trans. Fusion Technol., 25, 284 (1994).

10. A. M. MESSIAEN et al., Phys. Rev. Lett., 77, 2487 (1996).

11. F. DURODIÉ, M. VERVIER, and A. G. H. SIBLEY, Fusion Eng. Des., 56-57, 557 (2001).

12. G. WAIDMANN et al., Proc. 10th Int. Conf. Plasma Physics and Controlled Nuclear Fusion Research, London, 1984, Nucl. Fusion supplement, 1, 193 (1985).

13. A. M. MESSIAEN et al., Plasma Phys. Controlled Fusion, 28, 71 (1985).

14. P. E. VANDENPLAS et al., Plasma Phys Controlled Nucl. Fusion Res., 1, 485 (1986).

15. R. KOCH, V. P. BHATNAGAR, A. M. MESSIAEN, and D. VAN EESTER, Comput. Phys. Commun., 40, 1 (1986).

16. P. DESCAMPS et al., Plasma Phys. Controlled Fusion, 33, 1109 (1991).
17. A. M. MESSIAEN et al., Plasma Phys. Controlled Fusion, 31, 921 (1989).

18. R. VAN NIEUWENHOVE et al., Proc. 15th European Conf. Controlled Fusion and Plasma Heating, Dubrovnik, Croatia, Europhysics Conf. Abstracts, Vol. 12B, Part II, p. 778 (1988).

19. R. VAN NIEUWENHOVE and G. VAN OOST, J. Nucl. Mater. 162, 288 (1989).

20. R. VAN NIEUWENHOVE, G. VAN OOST, and P. E. VANDENPLAS, Fusion Eng. Des., 12, 231 (1990).

21. R. A. MOYER et al., J. Nucl. Mater., 176-177, 293 (1990).

22. A. M. MESSIAEN et al., Plasma Phys. Controlled Fusion, 32, 889 (1990).

23. B. UNTERBERG et al., "The Radiative Improved Mode in TEXTOR: Power Exhaust and Improved Confinement at High Density," Fusion Sci. Technol., 47, 187 (2005).

24. A. M. MESSIAEN et al., Plasma Phys. Controlled Fusion, 35, A15 (1993).

25. G. TELESCA et al., Proc. Europhysics Topl. Conf. Radiofrequency Heating and Current Drive of Fusion Devices, Brussels, Belgium, Europhysics Conf. Abstracts, Vol. 16E, p. 133 (1992).

26. R. KOCH et al., Plasma Phys. Controlled Fusion, 37, A291 (1995).

27. K. H. FINKEN et al., Phys. Rev. Lett., 73, 426 (1994).

28. R. KOCH et al., Fusion Eng. Des., 12, 15 (1990).

29. R. VAN NIEUWENHOVE, R. KOCH, and G. VAN OOST, LPPERM/KMS, Brussels Report No. 93 (1990).

30. R. VAN NIEUWENHOVE et al., Nucl. Fusion, 32, 1913 (1992).

31. R. VAN NIEUWENHOVE, R. KOCH, and G. VAN OOST, in Proc. 21 st Conf. Controlled Fusion and Plasma Physics, Montpellier, Europhysics Conf. Abstracts, Vol. 18B, Part II, p. 1036 (1994).

32. A. M. MESSIAEN, F. DURODIÉ, A. LYSSOIVAN, M. VERVIER, P. DUMORTIER, and R. KOCH, in Proc. 29th Conf. Plasma Physics and Controlled Fusion, Montreux, Switzerland, Europhysics Conf. Abstracts, Vol. 26B, P-2.062 (2002).

33. K. H. FINKEN et al., Fusion Eng. Des., 37, 335 (1997).

34. F. DURODIE et al., Fusion Eng. Des., 66-68, 509 (2003).

35. A. I. LYSSOIVAN et al., Proc. 22nd Conf. Controlled Fusion and Plasma Physics, Bournemouth, United Kingdom, Europhysics Conf. Abstracts, Vol. 19C, Part III, p. 341 (1995).

36. A. LYSSOIVAN et al., AIP Conf. Proc., 595, 146 (2001).

37. A. I. LYSSOIVAN et al., Proc. 2nd Europhysics Topl. Conf. Heating and Current Drive of Fusion Devices, Brussels, Belgium, Europhysics Conf. Abstracts, Vol. 22A, p. 85 (1998).

38. R. KOCH et al., Proc. 26th Conf. Controlled Fusion and Plasma Physics, Maastricht, The Netherlands, Europhysics Conf. Abstracts, Vol. 23J, p.745 (1999). 